\title{
Desafíos de la educación superior. Consideraciones sobre el Ecuador
}

\section{Challenges of higher education. Considerations about Ecuador}

Ruiz Gutiérrez Lourdes

Universidad Internacional del Ecuador, Ecuador

Torres Martínez Gisela

Universidad Central del Ecuador, Ecuador

García Céspedes Damarys

Universidad Técnica Luis Vargas Torres de Esmeraldas, Ecuador

Autor para correspondencia: 1ruiz@uide.edu.ec, gtorres@uce.edu.ec, damarys.garcia@utelvt.edu.ec

Fecha de recepción: 14 de Octubre de 2017 - Fecha de aceptación: 15 de Diciembre de 2017

Resumen: El presente trabajo tiene como objetivo analizar el sistema de educación superior ecuatoriano contemporáneo a partir de una metodología fundamentada en métodos de información, análisis y síntesis. Los métodos empleados se basan en una reseña histórica de las escuelas de pensamiento mundial y su influencia en la realidad latinoamericana; a partir de la búsqueda documental de fuentes bibliográficas y un estudio de caso de la universidad ecuatoriana. Se concluye con el planteamiento de un modelo enseñanza-aprendizaje basado el empleo de instrumentos legales vigentes y el diagnóstico de la problemática o nodos críticos del sistema de educación superior en el Ecuador y conclusiones que pueden aportar al perfeccionamiento del sistema de educación superior en nuestro país.

Palabras claves: educación superior; Ecuador; desafíos

\begin{abstract}
The present work takes as a target to analyze the Ecuadoran higher education system contemporary from a methodology based on methods of information, analysis and synthesis. The used methods are based on a historical critique of the schools of world thought and its influence in the Latin-American reality; from, documentary search of bibliographical sources and a case study of the Ecuadorian university. There concludes with the exposition of a model education - learning based the employment of current legal instruments and the diagnosis of the problems or critical nodes of the system of higher education in the Ecuador and conclusions that can reach port to the improving of the system of higher education in our country.
\end{abstract}

Key Words: higher education; Ecuador; challenges 


\section{Introducción}

Los sistemas de educación en todos los niveles y grados de enseñanza, constituyen uno de los factores más importantes del desarrollo y evolución de los países y sociedades tradicionales en su evolución y desarrollo sociocultural. No obstante en el contexto latinoamericano existió una influencia histórica heredada del pasado colonial, donde se aprecia que el modelo educativo de América Latina tiene sus antecedentes históricos copiados de los códigos educativos europeos.

Se plantea por Quijano (2000), que Europa se hizo el centro de la elaboración intelectual de la experiencia colonial/ moderna del conjunto del capitalismo. El resultado fue el eurocentrismo, una perspectiva de conocimiento tributaria por igual de las necesidades capitalistas de desmitificación del pensamiento sobre el universo y de las necesidades del blanco de legitimar y perpetuar su dominación/explotación como superioridad natural. Eso incluía la apropiación de las conquistas intelectuales y tecnológicas de los pueblos colonizados.

A estas ideas se agrega por Walsh (2006) sobre esta temática que dicha colonialidad se extiende a los campos del ser -la deshumanización y trato de no-existencia de algunos grupos-, y del saber -el posicionamiento del eurocentrismo y occidentalismo como modelos únicos del conocimiento, descartando las culturas indígenas autóctonas como intelectuales y como productores del conocimiento.

En la obra "La arqueología del saber", del filósofo francés Michel Foucault (1969) sobre los sistemas de pensamiento y conocimiento ("epistemes" o "formaciones discursivas") plantea que los mismos están regidos por reglas, de los sujetos individuales y que determinan los límites de pensamiento en un lugar y período dados, la noción de observación y su poder de cambiar el comportamiento de los individuos; con una visión eurocéntrica.

Los postulados filosóficos de Emmanuel Lévinas (1993), plasmados en su libro "ElTiempo-y-El-Otro", reconocen el sentido de la ética, la reconstrucción del pensamiento ético que sustenta que el conocimiento constituye una estrategia de apropiación, de dominación, presente fundamentalmente en la relación del hombre con el hombre y propone pensar de otro modo esta relación; que la hace incapaz de ser dominado.

El teórico argentino Daniel Schugurensky (1998) afirma que el tema de la globalización de la economía, y la mercantilización de la cultura se refleja en las limitaciones en el acceso a las universidades lo cual provoca una reducción en la diversidad social o étnica de los estudiantes y la proliferación de instituciones de segunda clase, generándose dos, tres o más niveles de calidad dentro del sistema.

Por otra parte la filósofa alemana Hannah Arendt (1951) en su obra "Los orígenes del totalitarismo", expande el concepto marxista del imperialismo con la dimensión del racismo y critica la reducción de la discusión sobre el capitalismo a puras cuestiones económicas. El móvil político del imperialismo es el intento de dividir la humanidad en «razas de señores y esclavos», en «negros y blancos». 
El insigne teórico brasileño de la educación del siglo XX, .Paulo Freire expresó: "no estamos en contra de vida burguesa, sino en contra de los excesos de pobreza". En "Pedagogía del oprimido" (1970), realiza la crítica a un modelo pedagógico de concibe a los estudiantes como recipientes en los cuáles será depositado el saber. Freire agrega que los docentes deben asumir una postura revolucionaria, para concientizar a las personas de la ideología opresora, y el propósito de la liberación de las clases oprimidas.

Según Aparicio (2011), el carácter regresivo de la política económica global y local se instituye simultáneamente junto a múltiples procesos de complejización social originado por las transformaciones estructurales de la vida pública, privada, civil, política, cotidiana y subjetiva gestadas en los últimos dos lustros en Argentina y Latinoamérica.

Hay estudios que muestran las condiciones, las posibilidades y las limitaciones de los países y los sistemas educativos de esta área, pero en un movimiento de tanta envergadura conviene tomar muy en cuenta las directrices, los procesos y los instrumentos que han sido usados con éxito en otras sociedades del mundo actual (Díez, 2002).

Jose Trainier (2013) expone que un ejemplo fue la crisis del año 2001 en Argentina y el fin de Neoliberalismo en América Latina donde el estado debió ocupar sus recursos para ir a los que más necesitan, desde una mirada de la política pública no de política partidaria. También dicho autor plantea analizar las articulaciones y modos de proyección en el espacio social y académico de la etapa neoliberal.

Plantea Trainier (2017) que hay teorías que hablan de que las Escuelas ya no sirven, que son aburridas, que hay que ampliar y rediseñar las estructuras. Al negar a las Escuela se niega al Estado. El docente es el único representante del Estado que puede ingresar a los barrios más pobres y olvidados. Agrega dicho autor que la educación es el primer bastión del cual se va a encargar la política pública. La educación trabaja con vínculos y existencias no con cosas, el impacto de las acciones tomará varios años.

Desde el año 1995, fue elaborado el "Documento de política para el cambio y el desarrollo en la educación superior. Resumen ejecutivo" de la UNESCO -y el Centro Regional para la Educación Superior en América Latina y el Caribe (CRESesALC) donde se definieron en esa época de finales de los noventa, las siguientes tendencias en la evolución reciente de la educación superior a escala mundial:

1. Expansión cuantitativa dada por: aumento del número y diversificación de los estudiantes; incremento del número de docentes; y la multiplicación de Instituciones de Educación Superior (IES).

2. Diversificación de las estructuras institucionales, los programas y las forman de estudio: es una de las tendencias mejor recibidas y debería ser apoyada para garantizar la calidad de instituciones y programas, la equidad respecto al acceso y la preservación de la misión y función de la educación superior.

3. Restricciones financieras: la correlación entre inversión en educación y el nivel de un país está bien establecido, por lo que es preocupante la tendencia que se observa a una disminución de los aportes estatales. 
A más de dos décadas después, se puede apreciar que las tendencias anunciadas en 1995 por la UNESCO están vigentes aún en Latinoamérica, sobre todo en la insuficiente inversión en las universidades y el escaso apoyo de los gobiernos. Por otra parte se señala por la UNESCO (2015), en el Foro Mundial sobre Educación celebrado en el año 2015, que la capacidad de aprendizaje a lo largo de toda la vida dentro de una educación de calidad es un tema priorizado que necesita un cambio de modelo que nos aleje de las ideas de enseñanza y capacitación y se aproxime a las de aprendizaje, de una instrucción transmisora de conocimientos a un aprendizaje para el desarrollo personal, y de una adquisición de competencias especiales a un descubrimiento de más amplio espectro y la liberación y el dominio del potencial creador. Este cambio es necesario en todos los niveles y en todas las modalidades de la educación, tanto en la educación formal como en la no formal y la informal.

Sin embargo aún persisten deficiencias a resolver según lo planteado por Fernández y Pérez (2016): "la necesidad para América Latina de continuar la expansión de sus sistemas de educación superior, fortalecer su capacidad científica y tecnológica y sostener en el largo plazo un esfuerzo incremental de financiamiento público que revierta los efectos de la fuerte desinversión educativa histórica en la región y que asegure un balanceado desarrollo del sistema universitario".

Sin considerar los intereses políticos, es necesario que la educación superior tenga el desafío de asumir el reto del exponencial crecimiento de la ciencia y las tecnologías, y priorizar los procesos de aprendizaje a fin de estimular la creatividad del sujeto que aprende y no en función de lo que el sujeto que enseña. La universidad debe enseñar a pensar, ejercitar el sentido común y dar rienda suelta a la imaginación creadora

\section{Consideraciones sobre la Educación Superior Ecuatoriana}

El Sistema de Educación Superior en el Ecuador se encuentra en una etapa de cambios para la mejora de los indicadores de calidad en las instituciones de educación superior y por consiguiente a su consolidación.

Según Ramírez (2013): “ volver propio un sistema cognitivo, implica construir un patrón de especialización sin desposesión ecológica y radicalmente democrático, además de construir también instituciones (redes) de inteligencia social, que canalicen la emancipación ciudadana desde sus derechos, necesidades y potencialidades, y que viabilicen el proyecto inconcluso, plurinacional e intercultural, llamado Ecuador, en el marco de la integración latinoamericana.

En estas ideas es importante resaltar la vinculación necesaria del sistema de educación superior ecuatoriano con las universidades latinoamericanas públicas y también privadas para la conformación de redes de conocimientos dentro de las especificidades propias del modelo nacional.

No obstante es importante lo señalado por Zabala (2015) que señala el modelo como "centralista burocrático para la determinación de los objetivos de política y los medios empleados" en donde se priorizan intereses políticos que preestablecen herramientas para estos fines. 
Los principios del Sistema de la Educación Superior, se establecen en la Constitución de la República del Ecuador. El Artículo 29 establece que:

“el Estado garantizará la libertad de enseñanza, la libertad de cátedra en la educación superior, y el derecho de las personas de aprender en su propia lengua y ámbito cultural. Además el Artículo 52 indica la autonomía responsable, cogobierno, igualdad de oportunidades, calidad, pertinencia, integralidad y autodeterminación”.

Además se emite la Ley Orgánica de Educación Superior (LOES), que define en el Artículo 3, que:

"la educación superior de carácter humanista, cultural y científica constituye un derecho de las personas y un bien público social, que de conformidad con la Constitución de la República, responderá al interés público y no estará al servicio de intereses individuales y corporativos".

- En el Artículo 8 de la propia LOES, se establecen los fines de la Educación Superior:

- Aportar al desarrollo del pensamiento universal, al despliegue de la producción científica y a la promoción de las transferencias e innovaciones tecnológicas;

- Fortalecer en las y los estudiantes un espíritu reflexivo orientado al logro de la autonomía personal, en un marco de libertad de pensamiento y de pluralismo ideológico;

- Contribuir al conocimiento, preservación y enriquecimiento de los saberes ancestrales y de la cultura nacional;

En el año 2013; se emite el Reglamento de Régimen Académico, amparado en el Artículo 166 de la Ley Orgánica de Educación Superior (LOES). El régimen académico se organiza a partir de los niveles de formación de la educación superior, la organización de los aprendizajes, la estructura curricular y las modalidades de aprendizaje o estudio y define las referencias epistemológicas y pedagógicas de las carreras y programas que se imparten. (Consejo de Educación Superior, 2014).

Para entender el cambio respecto a los proyectos educativos curriculares, es importante reconocer las tensiones o nodos críticos que el Sistema de Educación Superior Ecuatoriano ha atravesado en la última década, donde se evidencia una desarticulación entre la academia y la investigación científica. Dichos nodos se evidenciaron como regularidades diagnosticadas en las universidades ecuatorianas, los cuales reflejaron los problemas o nodos críticos se representan por Elizabeth Larrea (2013), en la Figura 1 y se determinan los problemas siguientes:

- El desarrollo de procesos de admisión que aseguren la calidad en los ingresos universitarios.

- La compatibilización entre el perfil de egreso del bachillerato y el perfil de ingreso de las IES.

- Los profesores de la los primeros años sin la preparación suficiente en los campos relacionados con los nuevos horizontes epistemológicos. 
- No existen programas de apoyo y acompañamiento pedagógico durante los primeros años dela formación profesional.

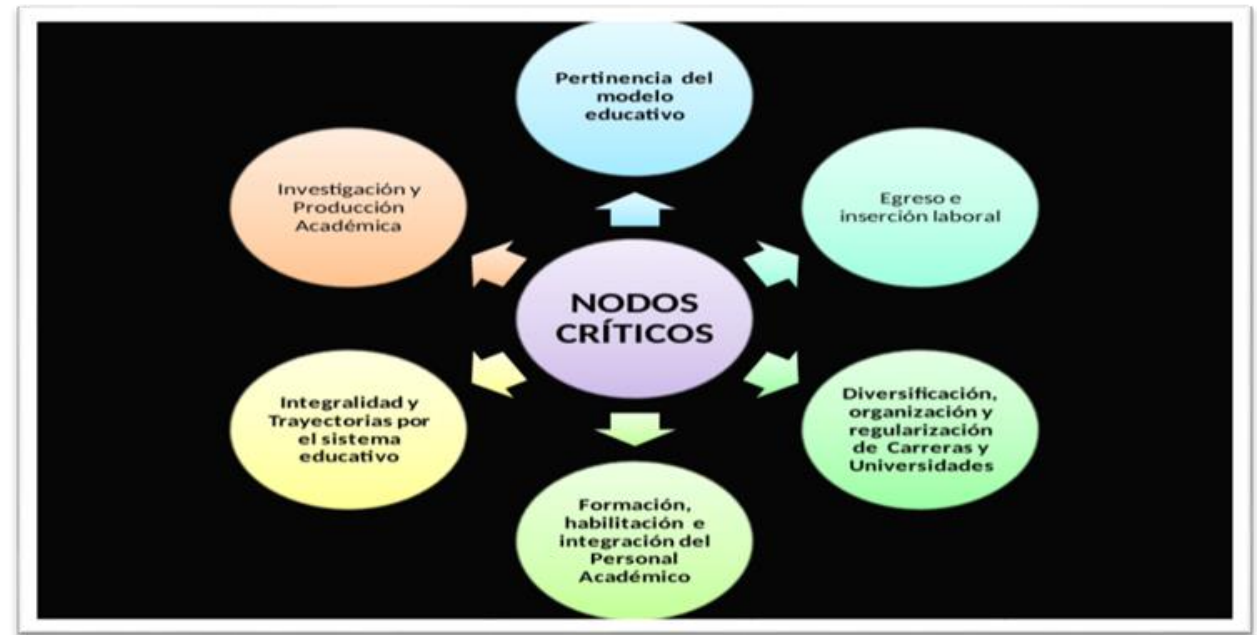

Figura 1. Nodos críticos que el Sistema de Educación Superior Ecuatoriano (Larrea, 2013).

Se presenta en la Figura 2, una propuesta de modelo educativo que puede servir como referente en el nuevo sistema de educación superior del Ecuador:
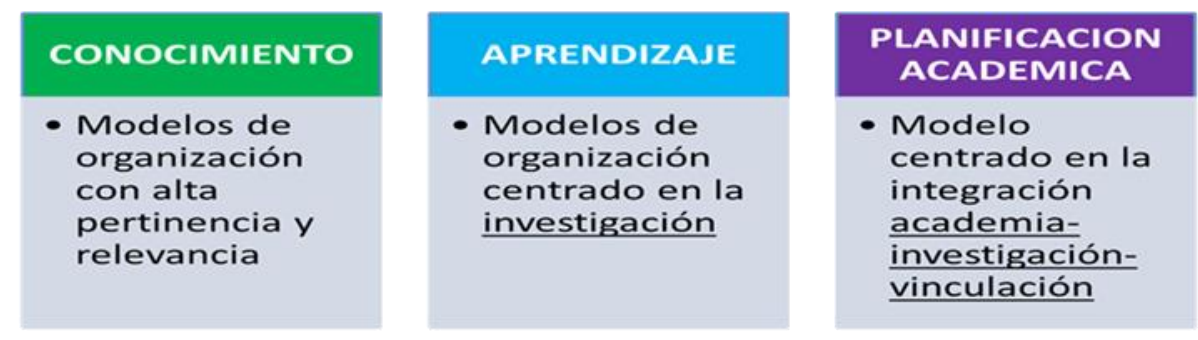

Figura 2. Modelo de conocimiento-aprendizaje-planificación académica

El modelo anterior está basado en la relación del conocimiento- aprendizaje y planificación académica con el eje de integración de la investigación científica y la vinculación de saberes con la sociedad, ya que se requiere aplicar una interacción de los conocimientos, donde la investigación, y la enseñanza-aprendizaje sean los protagonistas activos del proceso metodológico en las universidades del Ecuador.

Con respecto a la calidad del sistema universitario es necesario definir el concepto que según Tapia (2013) comprende un conjunto de estrategias (políticas, acciones, y procedimientos) que apuntan al control, la garantía y la promoción de la calidad de la institución en todos sus niveles para asegurar una mejora progresiva con miras a la excelencia.

En el sistema de educación superior ecuatoriano la calidad se constituye en un principio que consiste en la búsqueda constante y sistemática de la excelencia, la pertinencia, producción óptima, transmisión del conocimiento y desarrollo del pensamiento mediante la autocrítica, la crítica externa y el mejoramiento permanente (LOES, 2010). 
Autores como Moreira, Fleitas, Veliz, y Vinces, (2016), enfatizan acertadamente en que lo señalado en la LOES está enfocado a la visión de la calidad lograda si los estándares son alcanzados; y a la calidad como el grado en el cual la institución, de conformidad con su misión, ha alcanzado sus objetivos establecidos.

El modelo vigente de evaluación del Ecuador se organiza de acuerdo a los criterios siguientes: Organización, Academia, Investigaciones, Vinculación con la sociedad, Recursos e infraestructura y Estudiantes; y se centra en los resultados de los objetivos institucionales, pero no se enfoca en los indicadores de los procesos para alcanzarlos, dado que los patrones evalúan resultados y no el cómo se obtiene; y debe ser objeto de perfeccionamiento mediante la investigación científica (Veliz, 2018).

Resulta importante la necesidad de mejorar los sistemas de calidad en las universidades ecuatoriana entendiendo que lo académico no solo es docencia sino también investigación; vinculación con la sociedad y gestión universitaria.

\section{Conclusiones}

En un mundo como el actual, caracterizado por un cambio incesante e inesperado, y por una creciente globalización, el paradigma clásico de una educación tradicional y casi inmutable no resulta muy congruente con los nuevos escenarios y demandas sociales, y científicas, tanto actuales como futuras.

Si se considera que, cada vez más, importantes investigaciones coinciden en afirmar que «ninguna sociedad actual es superior a sus universidades», resulta evidente que un instrumento esencial del progreso y el desarrollo es la universidad

El Sistema de Educación Superior del Ecuador ha ido evolucionando de forma positiva para lograr la estrecha vinculación del modelo del conocimiento- aprendizaje y planificación académica con el eje de integración de la investigación científica y la vinculación de saberes con la sociedad.

Se debe mejorar los patrones de calidad internacional mediante la estrecha vinculación de los aspectos docentes con los de investigación; vinculación con la sociedad y gestión universitaria que es lo que constituye verdaderamente el indicador académico y no centrarse en la docencia como significado de la academia, que es el todo.

\section{Bibliografía}

Aparicio Pablo (2011). Crisis de la reforma educativa argentina en un contexto neoliberal. Los hándicaps de la inclusión educativa y laboral juvenil. Revista Electrónica Actualidades Investigativas en Educación. Universidad de Costa Rica.

Arendt Hannah (1951). Los orígenes del totalitarismo. New York. Harcourt Brace Jovanovich, Publishers, [1973]. 
Asamblea Constituyente de Ecuador (2008). Constitución Política de la República del Ecuador. Montecristi.

Casas Armengol, Miguel (2005). Nueva universidad ante la sociedad del conocimiento. RUSC. Universities and Knowledge Society Journal, http://www.redalyc.org/html/780/78020102/.

Consejo de Educación Superior (2015). Reglamento de Régimen Académico RPC-SO-18-No.2062015, 09 de abril de 2014, 17 de diciembre de 2014 y 06 de mayo de 2015. Ecuador.

Díez Hochleitner R. (2002). Aprender para el futuro. Documento básico de trabajo. Madrid: Fundación Santillana.

Fernández Lamarra, Pérez Centeno (2016). La educación superior latinoamericana en el inicio del nuevo siglo. Situación, principales problemas y perspectivas futuras. Revista Española de Educación Comparada. Número 27-2016. Madrid, PP. 123-148

Foucault, Michel (1969). La arqueología del saber. Buenos Aires: Siglo XXI ed. 1970.

Freire Paulo (1970). 1970: Pedagogía del Oprimido. Montevideo: Tierra Nueva, 1970.

Larrea Elizabeth (2012). El currículo genérico de la educación superior desde la complejidad sistémica. Consejo de Educación Superior del Ecuador. Quito:

Lévinas Emmanuel (1993). El tiempo y el otro. Ediciones Paidós Ibérica. ISBN 978-84-7509-8784.

LOES. (2010). Ley Orgánica de la Educación Superior. Suplemento del Registro Oficial No 298. Quito, Ecuador

Moreira, T. V., Fleitas, S., Veliz, V. \& Vinces, C. J. (2016). La vinculación con la sociedad en los patrones de calidad de universidades ecuatorianas e hispanoamericanas. Dilemas Contemporáneos. .Educación Políticas y Valores, 4(1): pp. 1-25

Quijano Aníbal (2000). El fantasma del desarrollo en América Latina. . Rev. Venezolana. De Economía. Y Ciencias Sociales, 2000, Vol. 6 No 2 (mayo-agosto), pp. 73-90

Ramírez René (2013). Tercera ola de transformación de la Educación Superior en Ecuador. Hacia la Constitucionalización de la sociedad del buen vivir. Dirección de Comunicación de la Secretaría Nacional de Educación Superior, Ciencia, Tecnología e Innovación (SENESCYT). Quito, pp.53

Schugurensky Daniel (1998). Reestructuración de la educación superior en la era de la globalización: ¿hacia un modelo heterónomo? Revista Educación, democracia y desarrollo en el fin de siglo, pp. 59-78 
Tapia, V. (2013). La gestión de la calidad de la educación universitaria: desafíos y posibilidades. ASPODEN (3), pp. 21-29

Tranier José (2017). Nuevos Escenarios de la Educación Contemporánea. Notas de clase del Doctorado con mención en Educación. Universidad del Rosario.

Tranier José (2013).Pensar la identidad institucional en contextos contemporáneos: sobre relatos, modelos, metáforas y abordajes. Rosario, Argentina, en los umbrales del Siglo XXI. Universidad de Costa Rica. DOI: https://doi.org/10.15517/revedu.v37i1.10637

Tünnermann C.; De Souza, M. (2003). Desafíos de la universidad en la sociedad del conocimiento. Cinco años después de la Conferencia Mundial sobre Educación Superior.

UNESCO (2014). Education Strategy 2014-2021.París. http://unesdoc.unesco.org/images/0023/002312/231288e.pdf

Veliz Briones (2018). Calidad en la Educación Superior. Caso Ecuador. Atenas, Revista Científico-Pedagógica. Vol.1. No. 41-2018. Universidad de Matanzas, PP. 165-180

Walsh Catherine (2006). Interculturalidad y (de) colonialidad: diferencia y nación de otro modo. Libro da Academia da Latinidades.: Textos \& Formas Ltda.

Zabala Tania (2015). Análisis del cambio de la política pública de Educación Superior en Ecuador, período 2008 - 2013. Tesis de maestría, Flacso Ecuador. 